\title{
Pancreatoduodenectomy within 2 weeks after endoscopic retrograde cholangio-pancreatography increases the risk of organ/space surgical site infections: a 5-year retrospective cohort study in a high-volume centre
}

\author{
Wensen Chen ${ }^{1,2 \#, ~ K a i ~ Z h a n g ~}{ }^{3,4 \#}$, Zhongheng Zhang ${ }^{5}$, Zipeng Lu ${ }^{3,4}$, Daoquan Zhang ${ }^{6}$, Juan Liu ${ }^{2}$, \\ Yue Yang ${ }^{2}$, Yinzhi Leng ${ }^{7}$, Yongxiang Zhang ${ }^{2}$, Weihong Zhang ${ }^{8}$, Kuirong Jiang ${ }^{3,4}$, Guihua Zhuang ${ }^{1}$, \\ Yi Miao ${ }^{3,4}$, Yun Liu ${ }^{9,10,11}$
}

${ }^{1}$ Department of Epidemiology and Biostatistics, School of Public Health, Xi'an Jiaotong University Health Science Center, Xi'an, China; ${ }^{2}$ Office of Infection Management, the First Affiliated Hospital of Nanjing Medical University, Nanjing, China; ${ }^{3}$ Pancreas Center, the First Affiliated Hospital of Nanjing Medical University, Nanjing, China; ${ }^{4}$ Pancreas Institute of Nanjing Medical University, Nanjing, China; ${ }^{5}$ Department of Emergency Medicine, Sir Run-Run Shaw Hospital, Zhejiang University School of Medicine, Hangzhou, China; ${ }^{6}$ Department of Endoscopy, the First Affiliated Hospital of Nanjing Medical University, Nanjing, China; ${ }^{7}$ Department of Infection, Nanjing Traditional Chinese Medicine Hospital, Nanjing, China; ${ }^{8}$ Office of Infection Management, Jiangsu Province Hospital \& Jiangsu Shengze Hospital, Suzhou, China; ${ }^{9}$ Department of Geriatrics Endocrinology, the First Affiliated Hospital of Nanjing Medical University, Nanjing, China; ${ }^{10}$ Department of Medical Informatics, School of Biomedical Engineering and Informatics, Nanjing Medical University, Nanjing, China; ${ }^{11}$ Institute of Medical Informatics and Management, Nanjing Medical University, Nanjing, China

Contributions: (I) Conception and design: W Chen, K Zhang, Z Lu, Y Liu, Y Miao, G Zhuang; (II) Administrative support: Y Liu, Y Miao; (III) Provision of study materials or patients: K Zhang, Z Lu, K Jiang; (IV) Collection and assembly of data: Z Zhang, D Zhang, J Liu, Y Yang, Y Leng, Y Zhang, W Zhang; (V) Data analysis and interpretation: W Chen, K Zhang; (VI) Manuscript writing: All authors; (VII) Final approval of manuscript: All authors.

"These authors contributed equally to this work.

Correspondence to: Guihua Zhuang, PhD. Department of Epidemiology and Biostatistics, School of Public Health, Xi'an Jiaotong University Health Science Center, No. 28, West Xianning Road, Xi'an 710049, China. Email: zhuanggh@mail.xjtu.edu.cn; Yun Liu, MD, PhD. Department of Geriatrics Endocrinology, the First Affiliated Hospital of Nanjing Medical University, School of Biomedical Engineering and Informatics, Nanjing Medical University, 101 Longmian Avenue, Jiangning District, Nanjing 211166, China. Email: 47437034@qq.com; Yi Miao, MD, PhD. Pancreas Center, the First Affiliated Hospital of Nanjing Medical University and Pancreas Institute of Nanjing Medical University, 101 Longmian Avenue, Jiangning District, Nanjing 211166, China. Email: miaoyi@njmu.edu.cn.

Backgroundk Organ/space surgical site infections (OSSI) after pancreaticoduodenectomy (PD) are not rare events. The role of diagnosis and treatment for pancreatic and biliary diseases with an endoscopic retrograde cholangio-pancreatography (ERCP) procedure is currently controversial. However, the ERCP procedure might play a role in surgical outcomes after PD.

Methods: We conducted a retrospective cohort study for patients who underwent PD in the First Affiliated Hospital with the Nanjing Medical University from 1st September 2012 to 31st January 2018. The relationship between ERCP exposure and OSSI after PD was analyzed by univariate and forward stepwise multivariate logistic regression model.

Results: Of the 1,365 patients who underwent PD, 136 developed OSSI (10.0\%). We found that ERCP exposure before PD (EEBPD) was significantly associated with an increased incidence rate of post-operative pancreas fistula (POPF) [24.2\% (23/95) vs. 14.9\% (189/1,270), risk ratio (RR) $=1.63,95 \%$ confidence interval (CI), 1.11-2.38, $\mathrm{P}=0.015$ ]. Hypertension, a higher level of preoperative low-density lipoprotein (LDL) and creatinine $(\mathrm{Cr})$ were associated with elevated risks of post-operative OSSI [adjusted odds ratio (Adj-OR) (95\% CI) were 1.59 (1.09-2.32), 1.70 (1.16-2.51), 1.99 (1.36-2.92)], whereas a preoperatively higher level of aspartate aminotransferase (AST) would decrease the risk [Adj-OR (95\% CI), 0.62 (0.42-0.91)]. Remarkably, 
EEBPD would significantly increase and more than double the OSSI risk [Adj-OR (95\% CI), 2.56 (1.464.47)] especially if it was within 14 days before surgery (Spearman $=-0.698, \mathrm{P}<0.001$ ).

Conclusions: ERCP, as an independent risk factor, significantly increased the risk of post-operative OSSI after PD if it is performed within 14 days prior to surgery. Our findings would assist clinical decision-making, and improve OSSI control and prevention.

Keywords: Endoscopic retrograde cholangio-pancreatography (ERCP); pancreaticoduodenectomy (PD); organ/ space surgical site infections (OSSI); logistic regression

Submitted Nov 13, 2020. Accepted for publication May 18, 2021.

doi: 10.21037 /gs-20-826

View this article at: https://dx.doi.org/10.21037/gs-20-826

\section{Introduction}

The CDC healthcare-associated infection (HAI) prevalence survey found that there were an estimated 157,500 surgical site infections (SSIs) [e.g., superficial incisional, deep incisional and organ/space surgical site infections (OSSI)] associated with inpatient surgeries in 2011 (1), and surgical-site infection was the leading cause of infection in hospitals (pooled cumulative incidence 5.6 per 100 surgical procedures), strikingly higher than the proportions recorded in developed countries (2). OSSI after surgery, are among the most common complications after bile duct, liver or pancreatic surgery [e.g., pancreaticoduodenectomy (PD)], and are associated with a prolonged length of stay in hospital and increased cost of treatment, imposing a significant economic burden on health care (3) and increasing the levels of hospital readmission (4). The occurrence of OSSI was adversely associated with longterm survival (5). PD is a standard of care for patients with malignant or benign disease of the pancreatic head or periampullary region $(6,7)$. PD is one of the most difficult techniques for gastroenterological surgeons, with long operation times, complicated gastrointestinal reconstruction procedures, and high morbidity rates (8). Postoperative SSI is more common after PD secondary to the extensive physiologic alterations (fluid shifts, blood loss, and systemic vasodilation) and multiple enteric anastomoses associated with the procedure. Even at high-volume tertiary referral centres, the incidence of post-PD wound infection is estimated to be approximately $10 \%$ to $17 \%$ (9-11).

An endoscopic retrograde cholangio-pancreatography (ERCP) procedure was sometimes applied to help identify the pancreaticobiliary limb or aiding in the visualisation of pancreatic and/or biliary anastomoses in patients with a $\mathrm{PD}$ resection (12). In patients awaiting PD, preoperative biliary drainage with ERCP was occasionally used to reduce bilirubin. However, the role of ERCP is being challenged as it is thought to increase infection and morbidity (13-15). The aim of this cohort study was to investigate the risks of preoperative ERCP procedure and OSSI for patients following PD.

We present the following article in accordance with the STROBE reporting checklist (available at https://dx.doi. org/10.21037/gs-20-826).

\section{Methods}

\section{Study design and setting}

This retrospective analysis of anonymous data was approved by the Institutional Review Board of the First Affiliated Hospital of Nanjing Medical University (2018-SR-295). The study was conducted in accordance with the Declaration of Helsinki (as revised in 2013). And written informed consent was obtained from all the study participants. We conducted an observational cohort study for patients who underwent PD in the First Affiliated Hospital of Nanjing Medical University from 1st September 2012 to 31st January 2018 and 1,365 patients were included. We installed the software of Healthcare associated infection Surveillance Platform (HSP) in 2012 which linked to a clinical database to extract the patients' data of inspection, diagnosis and treatment, and early warning of HAI. All the patients' data were extracted from the HSP and each OSSI was judged by infection control personnel (ICP) and the patient's attending doctor.

\section{Definitions}

OSSI were defined according to the National Healthcare Safety Network (NHSN). OSSI had to meet the following 
criteria: (I) date of event occurred within 30-90 days after the NHSN operative procedure (where day $1=$ the procedure date); (II) involves any part of the body deeper than the fascial/muscle layers that are opened or manipulated during the operative procedure; (III) the patient has at least one of the following: (i) purulent drainage from a drain that is placed into the organ/ space [for example, closed suction drainage system, open drain, T-tube drain, computed tomography (CT)-guided drainage]. (ii) Organism(s) identified from fluid or tissue in the organ/space by a culture- or non-culture-based microbiologic testing method, which is performed for purposes of clinical diagnosis or treatment [for example, not active surveillance culture/testing (ASC/AST)]. (iii) An abscess or other evidence of infection involving the organ/ space that is detected as the result of a gross anatomical or histopathologic examination, or imaging test evidence suggestive of infection; and (IV) meets at least one criterion for a specific organ/space infection site (these criteria are found in the Surveillance Definitions for Specific Types of Infections chapter) (CDC NHSN, https://www.cdc.gov/ HAI/ssi/ssi.html, Accessed 10/6/2018).

ERCP exposure was defined as the patients undergoing ERCP procedure within 3 months before PD.

Pancreatic fistulas (PFs) were defined and classified consistent with ISGPS criteria (16) Extracted data included patient demographics (age, gender, smoking status, alcohol abuse), comorbidities (hypertension, diabetes, coronary heart disease, obstructive jaundice), preoperative laboratory tests [total protein (TP), albumin (ALB), low-density lipoprotein (LDL), high-density lipoprotein (HDL), alanine aminotransferase (ALT), aspartate aminotransferase (AST), alkaline phosphatase (ALP), creatinine (Cr), creatine kinase (CK), urea, fasting blood-glucose (Glu), total bilirubin (TB)], ERCP exposure before PD (EEBPD), duration of ERCP and PD, blood transfusion and length of hospitalisation (LOH). Superficial and deep SSIs were not included in the analysis because they have different risk profiles and should be analyzed separately.

\section{Statistical analysis}

Data are presented as median [interquartile range (IQR)] or number (percentage). Instead of excluding cases with some missing data, multiple imputations were carried out. Each missing piece of data was imputed five times with the 'predictive mean matching' (PMM) method containing all of the predictor variables and the median one of these five values were imputed as missing data. Imputing a missing value could make the best use of the entire medical records (17). Categorical variables were compared using the Pearson's chi-square test or Fisher's exact test. Nonparametric continuous variables were analyzed using the Mann-Whitney $U$ test. Univariate and forward stepwise multivariate logistic analyses were performed to analyse the independent risk factors (18). For the subgroup analyses, we performed 1:4 propensity score matching (PSM) using the nearest neighbor matching on 95 EEBPD case and 1,270 no-EEBPD patients to optimize balance of baseline characteristics for assessing the independent effect of EEBPD. The primary matching criteria included hypertation, TP, Cr, ALB, LDL, HDL, CK, ALP, Glu, whose distribution was statistically different between the EEBPD and no-EEBPD groups. Propensity scores were calculated using the logistic regression model. After 1:4 PSM, the analysis included 95 EEBPD case and 380 noEEBPD patients and the variables were not significantly different between these two groups. The imputation of missing values was made using the 'mice' package (19), and the nomogram was developed and validated using the ' $r m s$ ' package. The PSM was performed by 'MatchIt' package. The decision-making tree was analysed and drew using the 'rpart' and 'rpart.plot' packages. The above statistical analyses were performed using Stata SE, version 11.0 (StataCorp., USA) or/and R software, version 3.5.1 (https:// www.r-project.org/). All statistics were two-tailed, and statistical significance was accepted at the $\mathrm{P}<0.05$ level.

\section{Results}

A total of 1,365 patients, who underwent PD from $1^{\text {st }}$ September 2012 to $31^{\text {st }}$ January 2018 in the First Affiliated Hospital with Nanjing Medical University, were identified in this cohort study. An additional figure showed this in more detail (see Figure S1). Within this total, there were 95 patients with ERCP exposure (7.0\%) and 1,270 patients without ERCP exposure (93.0\%). Patients' age, gender, history of smoking, alcohol abuse, hypertension, diabetes mellitus, coronary heart disease, obstructive jaundice and blood transfusion were similar between the two groups (Table 1). Perioperative ALB was discrepant between the two groups as well and ERCP exposure patients had higher ALB [median (IQR): 39.4 (36.2-42.8) vs. 36.5 (34.1-40.1) g/L, respectively; $\mathrm{P}<0.001]$. The distribution of HDL, CK and Glu was also significantly discrepant in the ERCP and non-ERCP exposure group. We found that the patients under EEBPD 
Table 1 Baseline characteristics and outcomes of the study population

\begin{tabular}{|c|c|c|c|}
\hline Number of participants & ERCP exposure $(n=95)$ & Non-ERCP exposure $(n=1,270)$ & $P$ value \\
\hline Male, n (\%) & $59(61.1)$ & $771(60.7)$ & 0.788 \\
\hline Smoking status (ever), n (\%) & $18(18.9)$ & $180(14.2)$ & 0.202 \\
\hline Alcohol abuse (ever), n (\%) & $14(14.7)$ & $154(12.1)$ & 0.455 \\
\hline \multicolumn{4}{|l|}{ Preoperative, median (IQR) } \\
\hline TP, g/L & $66.0(61.5-70.6)$ & $65.2(58.6-68.9)$ & 0.032 \\
\hline ALB, $g / L$ & $39.4(36.2-42.8)$ & $36.5(34.1-40.1)$ & $<0.001$ \\
\hline LDL, mmol/L & $3.37(2.65-4.30)$ & $3.11(2.46-4.08)$ & 0.086 \\
\hline $\mathrm{HDL}, \mathrm{mmol} / \mathrm{L}$ & $0.97(0.74-1.25)$ & $0.83(0.66-1.03)$ & 0.001 \\
\hline ALP, U/L & $252.6(95.6-519.9)$ & $248.0(151.1-444.9)$ & 0.226 \\
\hline $\mathrm{Cr}, \mu \mathrm{mol} / \mathrm{L}$ & $61.2(52.2-71.2)$ & $60.7(50.2-74.4)$ & 0.617 \\
\hline CK U/L & $55.0(38.7-76.0)$ & $37.0(27.0-50.0)$ & $<0.001$ \\
\hline Urea $\mathrm{mmol} / \mathrm{L}$ & $4.68(3.74-5.85)$ & $4.71(3.88-6.30)$ & 0.319 \\
\hline Glu mmol/L & $5.87(5.17-7.05)$ & $5.42(4.82-6.85)$ & 0.004 \\
\hline $\mathrm{TB}, \mu \mathrm{mol} / \mathrm{L}$ & $30.8(11.9-168.1)$ & $29.9(14.4-115.0)$ & 0.945 \\
\hline \multicolumn{4}{|l|}{ Comorbidities, n (\%) } \\
\hline Hypertension & $27(28.4)$ & $386(30.4)$ & 0.686 \\
\hline POPF (B or C), n (\%) & $23(24.2)$ & $189(14.9)$ & 0.015 \\
\hline LOH, median (IQR), day & $25.0(18.0-31.5)$ & $19.0(16.0-26.0)$ & $<0.001$ \\
\hline
\end{tabular}

ERCP, endoscopic retrograde cholangio-pancreatography; IQR, interquartile range; TP, total protein; ALB, albumin; LDL, low-density lipoprotein; HDL, high-density lipoprotein; ALT, alanine transaminase; AST, alanine aminotransferase; ALP, alkaline phosphatase; Cr, creatinine; CK, creatine kinase; Glu, fasting blood-glucose; TB, total bilirubin; POPF, post-operative pancreas fistula; LOH, length of hospitalisation.

were significantly associated with an increased incidence rate of post-operative pancreas fistula (POPF) [23/95 (24.2\%) vs. $189 / 1,270$ (14.9\%), $\mathrm{P}=0.015]$ and the LOH [25.0 (18.0-31.5) vs. $19.0(16.0-26.0), \mathrm{P}<0.001]$. For all of these 95 patients with ERCP exposure, 61 (64.2\%) patients were taken for preoperative diagnosis, $22(23.2 \%)$ patients were taken for biliary drainage, 5 (5.3\%) patients were taken for stent placement and 7 (7.4\%) patients were taken for other causes.

\section{Risk factors of OSSI}

The overall incidence rate of OSSI was 10.0\% (136/1,365). We found that the incidence rate of OSSI in the ERCP exposure group was significantly increased compared 
to that in the non-ERCP exposure group $(20.0 \%$ vs. $9.21 \%, 19 / 95$ vs. 117/1,270, $\mathrm{P}<0.002)$. Logistic regression analysis identified a history of EEBPD and hypertension were significantly associated with increased risk of postoperative OSSI [adjusted odds ratio (Adj-OR) (95\% CI), $2.56(1.46-4.47)$ and 1.59 (1.09-2.32), respectively], and higher levels of preoperative LDL and Cr were significantly associated with higher risk of OSSI [Adj-OR (95\% CI), 1.70 (1.16-2.51) and 1.99 (1.36-2.92), respectively]. However, we found that a higher level of preoperative ALP would significantly decrease the OSSI risk [Adj-OR (95\% CI), 0.62 (0.42-0.91)] (Table 2). The nomogram for the prediction

Table 2 Risk factors for OSSI in this cohort study

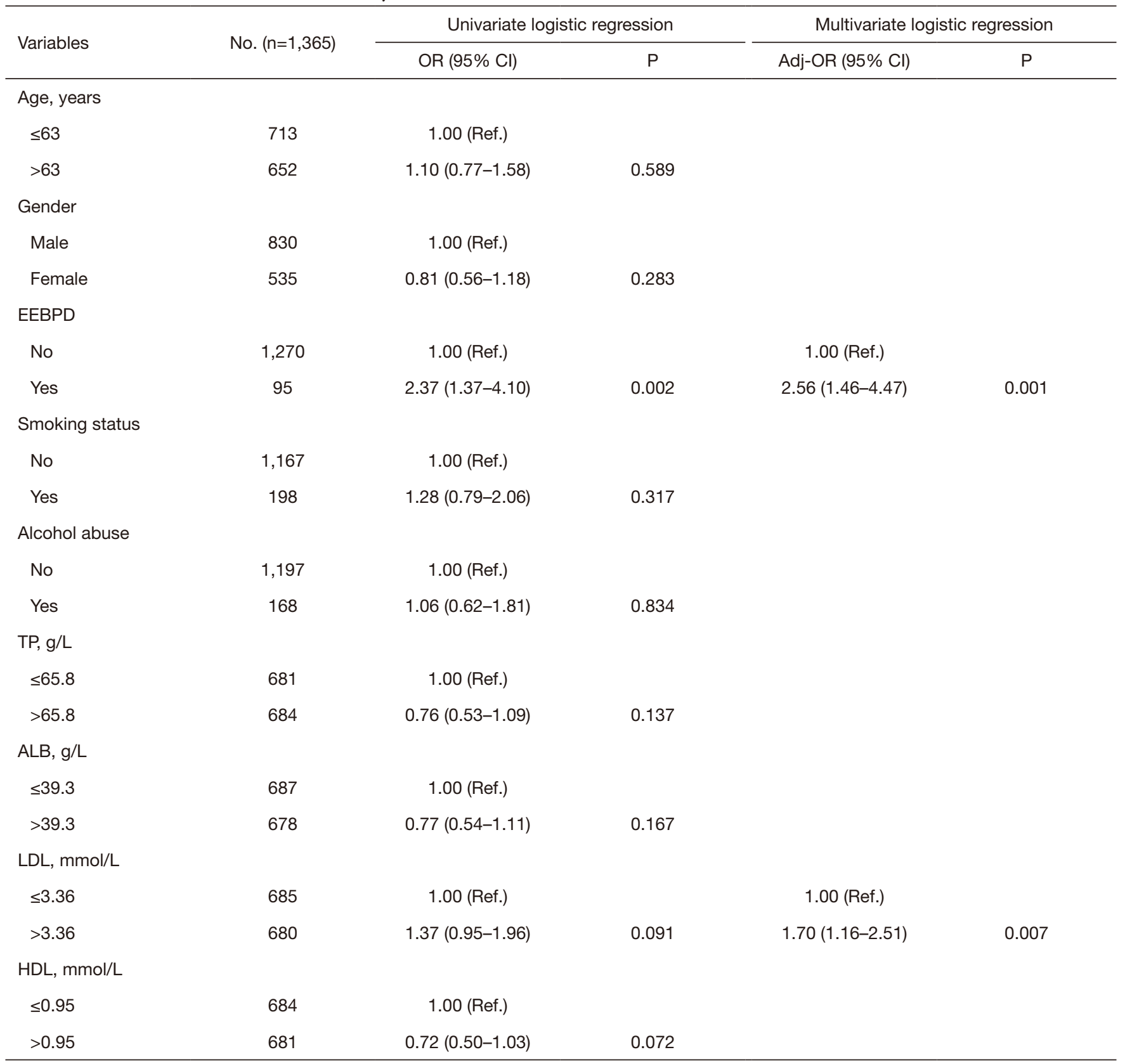

Table 2 (continued) 
Table 2 (continued)

\begin{tabular}{|c|c|c|c|c|c|}
\hline Variables & No. $(n=1,365)$ & \multicolumn{2}{|c|}{ Univariate logistic regression } & \multicolumn{2}{|c|}{ Multivariate logistic regression } \\
\hline \multicolumn{6}{|l|}{ ALT, U/L } \\
\hline$\leq 68.1$ & 683 & 1.00 (Ref.) & & & \\
\hline$>68.1$ & 682 & $0.91(0.63-1.30)$ & 0.589 & & \\
\hline$\leq 51.6$ & 683 & 1.00 (Ref.) & & & \\
\hline$>51.6$ & 682 & $0.82(0.57-1.17)$ & 0.276 & & \\
\hline \multicolumn{6}{|l|}{ ALP, U/L } \\
\hline$\leq 252.1$ & 683 & 1.00 (Ref.) & & 1.00 (Ref.) & \\
\hline$\leq 61.2$ & 677 & 1.00 (Ref.) & & 1.00 (Ref.) & \\
\hline$>61.2$ & 688 & $1.96(1.35-2.85)$ & $<0.001$ & $1.99(1.36-2.92)$ & $<0.001$ \\
\hline \multicolumn{6}{|l|}{ CK } \\
\hline$\leq 52.5$ & 677 & 1.00 (Ref.) & & & \\
\hline$>52.5$ & 688 & $1.32(0.92-1.89)$ & 0.137 & & \\
\hline \multicolumn{6}{|l|}{ Urea } \\
\hline$\leq 4.68$ & 589 & 1.00 (Ref.) & & & \\
\hline$>4.68$ & 676 & $1.53(1.07-2.21)$ & 0.021 & & \\
\hline \multicolumn{6}{|c|}{$\mathrm{TB}, \mu \mathrm{mol} / \mathrm{L}$} \\
\hline$>30.8$ & 680 & $1.01(0.70-1.44)$ & 0.965 & & \\
\hline \multicolumn{6}{|c|}{ Hypertension } \\
\hline No & 953 & 1.00 (Ref.) & & 1.00 (Ref.) & \\
\hline Yes & 412 & $1.57(1.08-2.27)$ & 0.017 & 1.59 (1.09-2.32) & 0.015 \\
\hline \multicolumn{6}{|l|}{ Diabetes } \\
\hline No & 1,148 & & & & \\
\hline Yes & 217 & $1.57(1.01-2.43)$ & 0.046 & & \\
\hline \multicolumn{6}{|c|}{ Coronary heart disease } \\
\hline No & 1,336 & 1.00 (Ref.) & & & \\
\hline Yes & 29 & $1.98(0.74-5.28)$ & 0.171 & & \\
\hline
\end{tabular}

Table 2 (continued) 
Table 2 (continued)

\begin{tabular}{|c|c|c|c|c|c|}
\hline Variables & No. $(n=1,365)$ & \multicolumn{2}{|c|}{ Univariate logistic regression } & \multicolumn{2}{|c|}{ Multivariate logistic regression } \\
\hline \multicolumn{6}{|c|}{ Obstructive jaundice } \\
\hline No & 816 & 1.00 (Ref.) & & & \\
\hline Yes & 549 & $0.81(0.55-1.17)$ & 0.256 & & \\
\hline \multicolumn{6}{|c|}{ Cholangitis } \\
\hline No & 1,171 & 1.00 (Ref.) & & & \\
\hline Yes & 194 & $1.16(0.71-1.90)$ & 0.557 & & \\
\hline
\end{tabular}

OSSI, organ/space surgical site infections; OR, odds ratio; Adj-OR, adjusted OR; CI, confidence interval; EEBPD, ERCP exposure before PD; ERCP, endoscopic retrograde cholangio-pancreatography; PD, pancreaticoduodenectomy; TP, total protein; ALB, albumin; LDL, low-density lipoprotein; HDL, high-density lipoprotein; ALT, alanine transaminase; AST, alanine aminotransferase; ALP, alkaline phosphatase; Cr, creatinine; CK, creatine kinase; Glu, fasting blood-glucose; TB, total bilirubin.

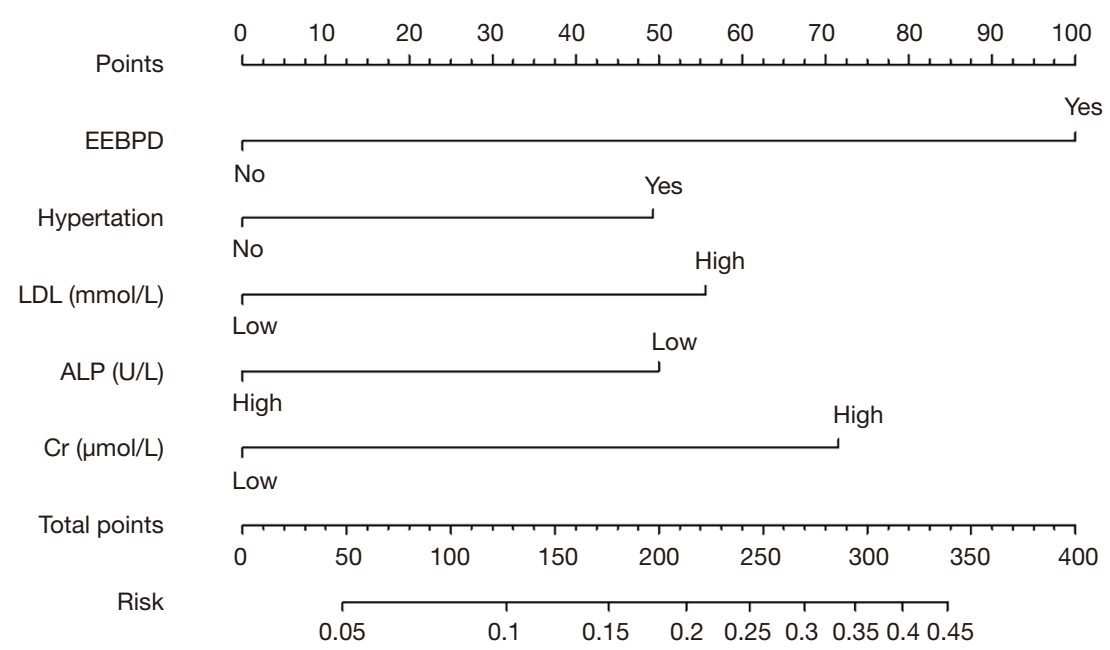

Figure 1 Nomogram for the risk prediction of OSSI. To use the nomogram, for an individual patient, the value is loaded on each variable axis (the $2^{\text {nd }}-6^{\text {th }}$ lines), and a line is drawn upward to determine the number of points received for each variable value (the $1^{\text {st }}$ line). The sum of these numbers is located on the total points axis (the $6^{\text {th }}$ line), and a line is drawn downward to the risk axes (the $8^{\text {th }}$ line) to determine the risk of OSSI. OSSI, organ/space surgical site infections; EEBPD, ERCP exposure before PD; ERCP, endoscopic retrograde cholangiopancreatography; PD, pancreaticoduodenectomy; LDL, low-density lipoprotein; ALP, alkaline phosphatase; Cr, creatinine.

of postoperative OSSI integrating all these independent predictors is presented in Figure 1.

\section{ERCP functional analysis and clinical decision}

Among 1,365 patients, 95 received ERCP before PD. $57.9 \%(55 / 95)$ ERCP procedure was conducted in our hospital. In our current cohort, we found that the OSSI rate was negatively correlated with the interval days between
ERCP and surgery (Spearman $\mathrm{r}=-0.698, \mathrm{P}<0.001)$. With further analysis, we found that 14 days between ERCP and PD might be the appropriate interval time for a lower OSSI rate with $25 \%$ reduction (Figure 2).

\section{Subgroup analyses of patients after propensity score-matching}

After propensity score-matching for hypertation, TP, Cr, 


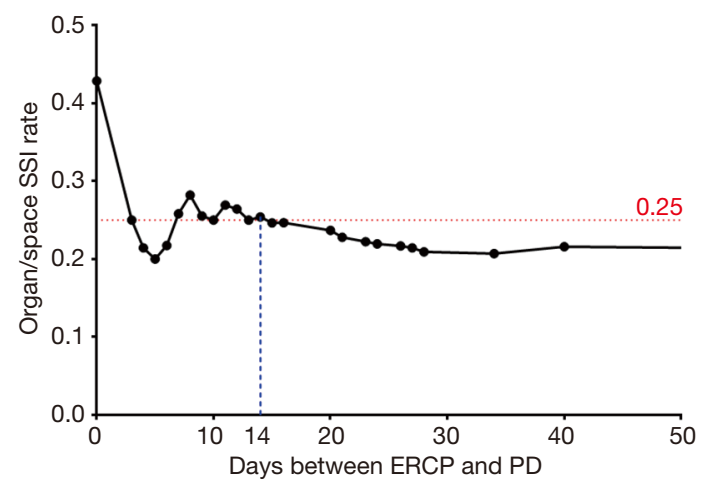

Figure 2 Appropriate time of ERCP for post-PD OSSI rate. The dotted red line presented the infection rate of $25 \%$ and the dotted blue line presented the inflection day for infection rate. ERCP, endoscopic retrograde cholangio-pancreatography; $\mathrm{PD}$, pancreaticoduodenectomy; OSSI, organ/space surgical site infections.

ALB, LDL, HDL, CK, ALP, Glu, there was no significant differences in these nine variables between patients with or without EEBPD (Table 3). Patients with EEBPD exhibited an increased the risk of SSI [OR, 2.29; 95\% CI, 1.23 to 4.28, $\mathrm{P}=0.009$ ] compared with those not receiving EEBPD after adjusted hypertation, Cr, LDL and ALP.

\section{Microbiology analysis of OSSI}

Among 144 isolations, the predominant microorganisms cultured from OSSI after PD were E. coli (34 episodes, $23.61 \%$, 34/144), K. pneumoniae (30 episodes, 20.83\%, 30/144) and E. faecium (19 episodes, 13.19\%, 19/144) (Figure 3). An additional table showed this in more detail (see Table S1).

\section{Discussion}

$\mathrm{PD}$ is one of the most stressful types of surgery for gastroenterological surgeons. The development of OSSI is a common complication after PD with the incidence of wound infection as high as $10 \%$ to $17 \%(3,9-11,20)$ and the incidence of OSSI was 48\% in Japan in 2012 (21). The postoperative OSSI for these patients poses a significant clinicalland economic burden in the field of surgery (22), often leading to extended length of stay, readmissions, and attendant increased costs (23). Therefore, reducing the incidence of OSSI is important; however, we know little about the risk factors for PD-related OSSI. For these reasons, we analyzed the incidence of OSSI following PD and tried to find a method to reduce the incidence of OSSI.

In this large volume teaching hospital, we performed a retrospective cohort study between 1st September 2012 and 31st January 2018 and the overall OSSI incidence rate was $10.0 \%$ for the patients undergoing PD. Sugiura and the group revealed several risk factors such as length of operation $>480 \mathrm{~min}$, main pancreatic duct (MPD) $\leq 3 \mathrm{~mm}$, body mass index $(\mathrm{BMI})>23.5 \mathrm{~kg} / \mathrm{m}^{2}$, semi-closed drain and $\mathrm{PF}$, that were associated with OSSI. The presence of a PF was the strongest risk factor for OSSI (21). Patients who develop a POPF have a higher risk of dying after surgery, and need to stay longer in the hospital (7). A PF after PD always occurs due to autolysis caused by activated trypsin, resulting in tissue damage around the pancreatic ductal anastomosis (23). A study from Scheufele found that the postoperative wound infections would be increased after preoperative biliary drainage (24) and there were fundamental differences in the biliary microbiome of patients with periampullary cancer who undergo preoperative biliary drainage and those who do not (25). In our study, we analyzed the risk factors associated with OSSI before PD. The EEBPD was the strongest risk factor for developing an OSSI (Adj$\mathrm{OR}=2.56$ ). The incidence of OSSI in patients with ERCP exposure was $20 \%$, which was over one time higher than that observed in patients without (9\%). Barreto et al. also found that preoperative ERCP and stenting may result in a significantly higher risk of SSIs while they were unable to document an association between the development of POPF and SSIs $(\mathrm{P}=0.308)$ (26). Besides, many studies had reported that preoperative biliary drainage is associated with positive intraoperative bile cultures (27-30), and accessing the biliary tract from the digestive tract is known to increase the risk of postoperative infectious complications (31-33). Results from $\mathrm{Wu}$ et al. Showed that ERBD was associated with an increased risk of intra-abdominal abscess (IAA) in patients undergoing PD (34). It's similar to SSI that Endoscopy, angiography, and/or exploratory laparotomy was associated with grade $\mathrm{B}$ or $\mathrm{C}$ hemorrhage $(23$ of $54,42.6 \%$ ) in post-PD patients (35). ERCP is a procedure that enables the physician to examine the pancreatic and bile ducts and it needs a small opening in the duodenum (ampulla) and a small plastic tube (cannula) is then passed through the endoscope and into this opening. The small opening might be the reason for increased risk. Interestingly, we also found that the risk of ERCP operation within 14 days of PD is associated with a higher risk of OSSI, which strongly suggested that this 
Table 3 Subgroup analyses of patients after propensity score-matching

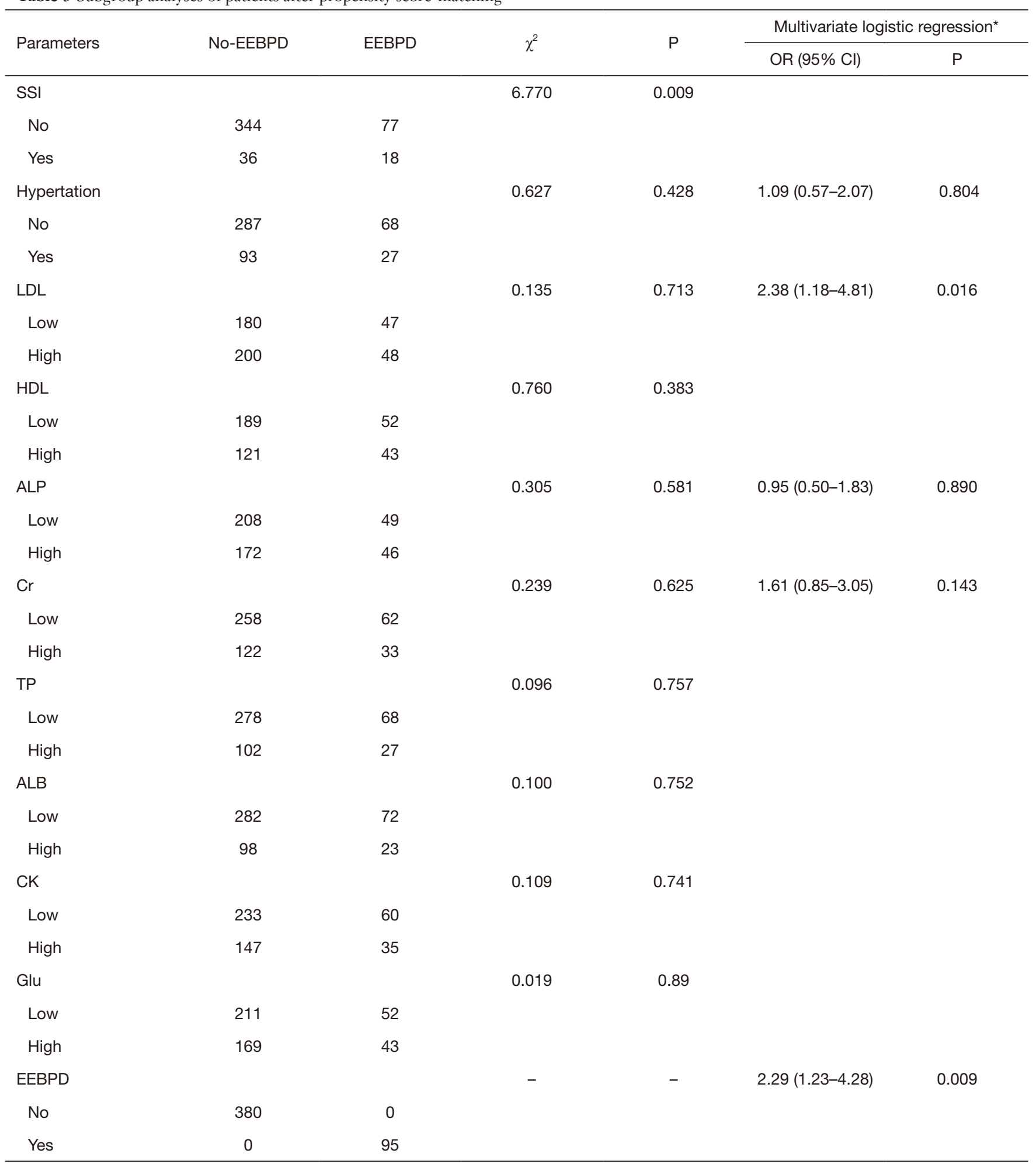

*, multivariate logistic regression analyses for PD-patients with or without OSSI after propensity score-matching. EEBPD, ERCP exposure before PD; ERCP, endoscopic retrograde cholangio-pancreatography; PD, pancreaticoduodenectomy; OR, odds ratio; Cl, confidence interval; SSI, surgical site infection; LDL, low-density lipoprotein; HDL, high-density lipoprotein; ALP, alkaline phosphatase; Cr, creatinine; TP, total protein; ALB, albumin; CK, creatine kinase; Glu, fasting blood-glucose. 


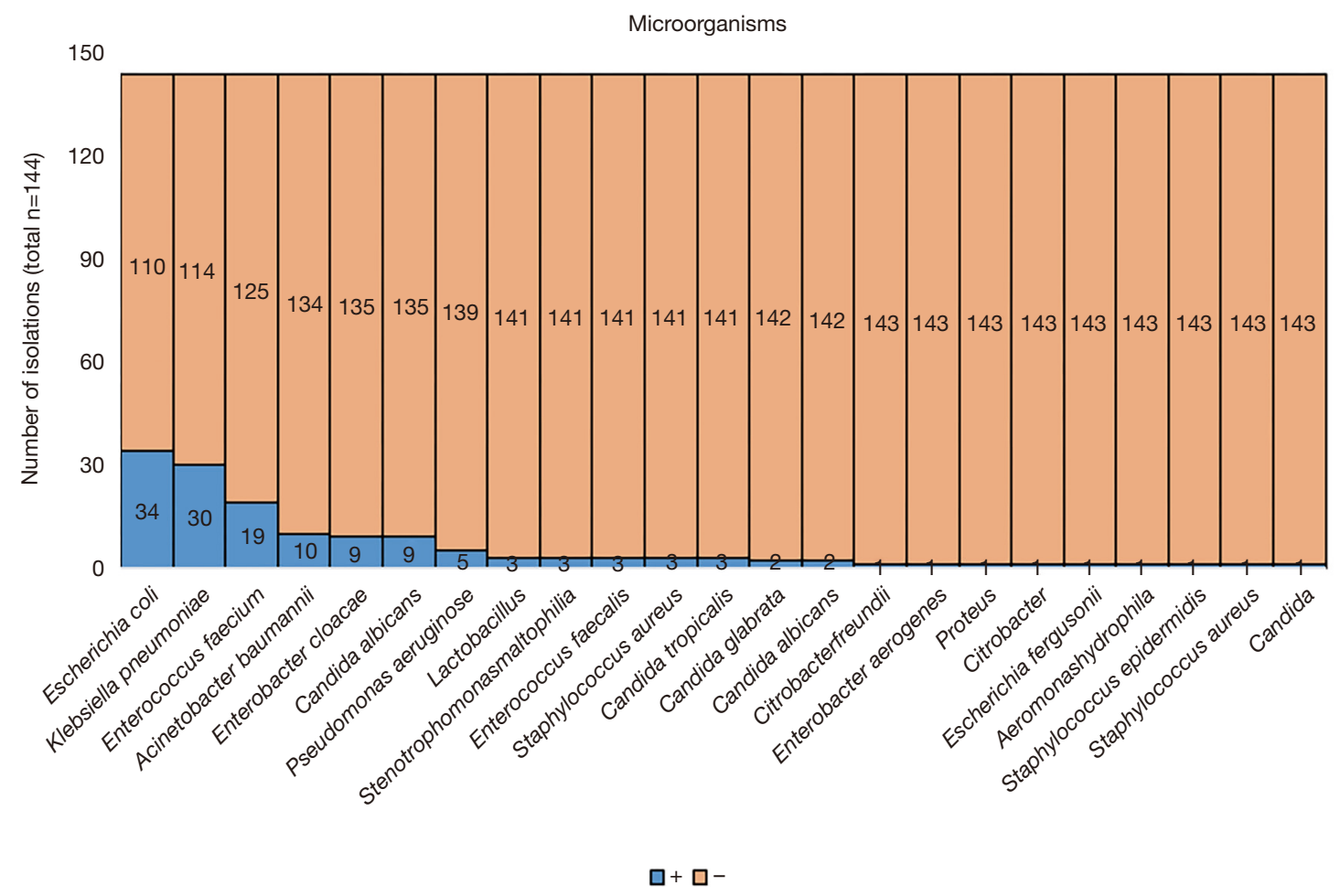

Figure 3 Microbiology analysis of OSSI. Each column represents a microorganism and the row represents the numbers of patients; orange represented uninfected and blue represented infected. OSSI, organ/space surgical site infections.

elective PD time should be delayed for at least 14 days without any contraindications.

When analyzing OSSI culture microorganisms, among 144 isolations, gram-negative bacteria were identified in most cases and the predominant microorganisms cultured from post-PD OSSI were E. coli, Kpneumoniae and E. faecium. The most frequently isolated bacteria were Enterococcus species (51\%), Klebsiella species (28\%), and Escherichia coli (27\%) (34). The change of the bacterial spectrum could be the selection of microorganisms through the use of antibiotics $(36,37)$, which could result in different microbiology profiles of the cultured bacteria.

We found that preoperative higher level of LDL and $\mathrm{Cr}$ was significantly with higher risk of OSSI while higher level of ALP would significantly decrease the OSSI risk. Our finding suggested that biomarkers in concentration could be predictors for SSIs. Delgado-Rodríguez et al. found that serum HDL-C and total cholesterol seem to be associated with the risk of nosocomial infection in surgical patients (38). Meanwhile, Mahdi et al. revealed that serum Cr concentration $\geq 2 \mathrm{mg} / \mathrm{dL}$ was significantly associated with SSI among those who underwent laparoscopic hysterectomy (39). Watanabe et al. consider that chronic kidney disease (serum $\mathrm{Cr}$ concentration $>1.2 \mathrm{mg} / \mathrm{dL}$ ) correlated with a higher risk of incisional SSI. Kidney disease may be a potential risk factor for abdominal infection (40). Based on these risk factors, we developed the nomogram to display the results and the decision-making tree to assist clinicians in decision-making.

Although the results were promising, this study had some shortcomings. Firstly, preoperative jaundice reduction was not performed routinely in our hospital, which resulted in a smaller proportion of preoperative ERCP. The total number of cases with preoperative ERCP was only 95, which might introduce some bias. In addition, this study was a retrospective study and several factors such as duct diameter and BMI were not available. All these results and more factors need to be validated by prospective studies.

\section{Conclusions}

Our analysis of data from a cohort study over 5 years identified that EEBPD would significantly increase risks of OSSI for patients following PD, especially within 2 weeks. 
We propose that the patients undergoing ERCP who are preparing for PD should be delayed for at least 2 weeks. Otherwise, a non-invasive procedure for diagnosis should be selected, such as CT or magnetic resonance cholangiopancreatography (MRCP). Hypertension, preoperative higher LDL and Cr would significantly increase OSSI risks while a preoperatively higher level of ALP was significantly associated with lower risks of OSSI. Based on our reported data, the predictive function of nomogram could be referred to lower the risks of OSSI for the patients following PD.

\section{Acknowledgments}

The wordings of the main text and figures/tables were checked by Quality Proofreading by PSUK Communications Ltd., https://qualityproofreading.co.uk/.

Funding: This work was supported by grants from the National Key Research \& Development Plan of Ministry of Science and Technology of the People's Republic of China (Grant No. 2018YFC1314900 and Grant No. 2018YFC1314901), the 2016 Industry Prospecting and Common Key Technology Key Projects of Jiangsu Province Science and Technology Department (Grant No. BE2016002-4), the Project of Jiangsu Provincial Department of Finance [(2018)0419, (2017)79], the 2016 Projects of Nanjing Science Bureau (Grant No. 201608003), and the Clinical Capability Improvement Project of Jiangsu Province Hospital (JSPH-MB-2020-10).

\section{Footnote}

Reporting Checklist: The authors have completed the STROBE reporting checklist. Available at https://dx.doi. org/10.21037/gs-20-826

Data Sharing Statement: Available at https://dx.doi. org/10.21037/gs-20-826

Peer Review File: Available at https://dx.doi.org/10.21037/ gs-20-826

Conflicts of Interest: All authors have completed the ICMJE uniform disclosure form (available at https://dx.doi. org/10.21037/gs-20-826). The authors have no conflicts of interest to declare.

Ethical Statement: The authors are accountable for all aspects of the work in ensuring that questions related to the accuracy or integrity of any part of the work are appropriately investigated and resolved. The study was conducted in accordance with the Declaration of (as revised in 2013). This study had been approved by the Ethical Review Committee of the First Affiliated Hospital of Nanjing Medical University (2018-SR-295) and informed consent was taken from all individual participants.

Open Access Statement: This is an Open Access article distributed in accordance with the Creative Commons Attribution-NonCommercial-NoDerivs 4.0 International License (CC BY-NC-ND 4.0), which permits the noncommercial replication and distribution of the article with the strict proviso that no changes or edits are made and the original work is properly cited (including links to both the formal publication through the relevant DOI and the license). See: https://creativecommons.org/licenses/by-nc-nd/4.0/.

\section{References}

1. Magill SS, Edwards JR, Bamberg W, et al. Multistate point-prevalence survey of health care-associated infections. N Engl J Med 2014;370:1198-208.

2. Allegranzi B, Bagheri Nejad S, Combescure C, et al. Burden of endemic health-care-associated infection in developing countries: systematic review and meta-analysis. Lancet 2011;377:228-41.

3. Fong ZV, McMillan MT, Marchegiani G, et al. Discordance between perioperative antibiotic prophylaxis and wound infection cultures in patients undergoing pancreaticoduodenectomy. JAMA Surg 2016;151:432-9.

4. Howard JD Jr, Ising MS, Delisle ME, et al. Hospital readmission after pancreaticoduodenectomy: a systematic review and meta-analysis. Am J Surg 2019;217:156-62.

5. Buettner S, Ethun CG, Poultsides G, et al. Surgical site infection is associated with tumor recurrence in patients with extrahepatic biliary malignancies. J Gastrointest Surg 2017;21:1813-20.

6. Cheng Y, Briarava M, Lai M, et al. Pancreaticojejunostomy versus pancreaticogastrostomy reconstruction for the prevention of postoperative pancreatic fistula following pancreaticoduodenectomy. Cochrane Database Syst Rev 2017;9:CD012257.

7. Topal B, Fieuws S, Aerts R, et al. Pancreaticojejunostomy versus pancreaticogastrostomy reconstruction after pancreaticoduodenectomy for pancreatic or periampullary tumours: a multicentre randomised trial. Lancet Oncol 
2013;14:655-62.

8. De Pastena M, Paiella S, Marchegiani G, et al. Postoperative infections represent a major determinant of outcome after pancreaticoduodenectomy: results from a high-volume center. Surgery 2017;162:792-801.

9. Winter JM, Cameron JL, Campbell KA, et al. 1423 pancreaticoduodenectomies for pancreatic cancer: a singleinstitution experience. J Gastrointest Surg 2006;10:1199210; discussion 1210-1.

10. Fernández-del Castillo C, Morales-Oyarvide V, McGrath D, et al. Evolution of the Whipple procedure at the Massachusetts General Hospital. Surgery 2012;152:S56-63.

11. Kent TS, Sachs TE, Callery MP, et al. The burden of infection for elective pancreatic resections. Surgery 2013;153:86-94.

12. Enestvedt BK, Kothari S, Pannala R, et al. Devices and techniques for ERCP in the surgically altered GI tract. Gastrointest Endosc 2016;83:1061-75.

13. Dorcaratto D, Hogan NM, Munoz E, et al. Is percutaneous transhepatic biliary drainage better than endoscopic drainage in the management of jaundiced patients awaiting pancreaticoduodenectomy? A systematic review and metaanalysis. J Vasc Interv Radiol 2018;29:676-87.

14. van der Gaag NA, Rauws EA, van Eijck CH, et al. Preoperative biliary drainage for cancer of the head of the pancreas. N Engl J Med 2010;362:129-37.

15. Fang Y, Gurusamy KS, Wang Q, et al. Meta-analysis of randomized clinical trials on safety and efficacy of biliary drainage before surgery for obstructive jaundice. Br J Surg 2013;100:1589-96.

16. Bassi C, Marchegiani G, Dervenis C, et al. The 2016 update of the International Study Group (ISGPS) definition and grading of postoperative pancreatic fistula: 11 years after. Surgery 2017;161:584-91.

17. Harrell FE Jr, Lee KL, Mark DB. Multivariable prognostic models: issues in developing models, evaluating assumptions and adequacy, and measuring and reducing errors. Stat Med 1996;15:361-87.

18. Zhang Z. Variable selection with stepwise and best subset approaches. Ann Transl Med 2016;4:136.

19. Zhang Z. Multiple imputation with multivariate imputation by chained equation (MICE) package. Ann Transl Med 2016;4:30.

20. Shinkawa H, Takemura S, Uenishi T, et al. Nutritional risk index as an independent predictive factor for the development of surgical site infection after pancreaticoduodenectomy. Surg Today 2013;43:276-83.
21. Sugiura T, Uesaka K, Ohmagari N, et al. Risk factor of surgical site infection after pancreaticoduodenectomy. World J Surg 2012;36:2888-94.

22. Santema TB, Visser A, Busch OR, et al. Hospital costs of complications after a pancreatoduodenectomy. HPB (Oxford) 2015;17:723-31.

23. Yamashita K, Sasaki T, Itoh R, et al. Pancreatic fistulae secondary to trypsinogen activation by Pseudomonas aeruginosa infection after pancreatoduodenectomy. J Hepatobiliary Pancreat Sci 2015;22:454-62.

24. Scheufele F, Schorn S, Demir IE, et al. Preoperative biliary stenting versus operation first in jaundiced patients due to malignant lesions in the pancreatic head: a meta-analysis of current literature. Surgery 2017;161:939-50.

25. Scheufele F, Aichinger L, Jäger C, et al. Effect of preoperative biliary drainage on bacterial flora in bile of patients with periampullary cancer. Br J Surg 2017;104:e182-8.

26. Barreto SG, Singh MK, Sharma S, et al. Determinants of surgical site infections following pancreatoduodenectomy. World J Surg 2015;39:2557-63.

27. Kobayashi S, Gotohda N, Nakagohri T, et al. Risk factors of surgical site infection after hepatectomy for liver cancers. World J Surg 2009;33:312-7.

28. Jethwa P, Breuning E, Bhati C, et al. The microbiological impact of pre-operative biliary drainage on patients undergoing hepato-biliary-pancreatic (HPB) surgery. Aliment Pharmacol Ther 2007;25:1175-80.

29. Sudo T, Murakami Y, Uemura K, et al. Specific antibiotic prophylaxis based on bile cultures is required to prevent postoperative infectious complications in pancreatoduodenectomy patients who have undergone preoperative biliary drainage. World J Surg 2007;31:2230-5.

30. Fujii T, Yamada S, Suenaga M, et al. Preoperative internal biliary drainage increases the risk of bile juice infection and pancreatic fistula after pancreatoduodenectomy: a prospective observational study. Pancreas 2015;44:465-70.

31. Cortes A, Sauvanet A, Bert F, et al. Effect of bile contamination on immediate outcomes after pancreaticoduodenectomy for tumor. J Am Coll Surg 2006;202:93-9.

32. Povoski SP, Karpeh MS Jr, Conlon KC, et al. Preoperative biliary drainage: impact on intraoperative bile cultures and infectious morbidity and mortality after pancreaticoduodenectomy. J Gastrointest Surg 1999;3:496-505.

33. Velanovich V, Kheibek T, Khan M. Relationship of 
postoperative complications from preoperative biliary stents after pancreaticoduodenectomy. A new cohort analysis and meta-analysis of modern studies. JOP 2009; 10:24-9.

34. Wu JM, Ho TW, Yen HH, et al. Endoscopic retrograde biliary drainage causes intra-abdominal abscess in pancreaticoduodenectomy patients: an important but neglected risk factor. Ann Surg Oncol 2019;26:1086-92.

35. Lu JW, Ding HF, Wu XN, et al. Intraabdominal hemorrhage following 739 consecutive pancreaticoduodenectomy: risk factors and treatments. J Gastroenterol Hepatol 2019;34:1100-7.

36. Müssle B, Hempel S, Kahlert C, et al. Prognostic impact of bacterobilia on morbidity and postoperative management after pancreatoduodenectomy: a systematic review and meta-analysis. World J Surg 2018;42:2951-62.

Cite this article as: Chen $\mathrm{W}$, Zhang $\mathrm{K}$, Zhang Z, Lu Z, Zhang D, Liu J, Yang Y, Leng Y, Zhang Y, Zhang W, Jiang K, Zhuang G, Miao Y, Liu Y. Pancreatoduodenectomy within 2 weeks after endoscopic retrograde cholangio-pancreatography increases the risk of organ/space surgical site infections: a 5-year retrospective cohort study in a high-volume centre. Gland Surg 2021;10(6):1852-1864. doi: 10.21037/gs-20-826
37. Kwon W, Jang JY, Kim EC, et al. Changing trend in bile microbiology and antibiotic susceptibilities: over 12 years of experience. Infection 2013;41:93-102.

38. Delgado-Rodríguez M, Medina-Cuadros M, MartínezGallego G, et al. Total cholesterol, HDL-cholesterol, and risk of nosocomial infection: a prospective study in surgical patients. Infect Control Hosp Epidemiol 1997;18:9-18.

39. Mahdi H, Goodrich S, Lockhart D, et al. Predictors of surgical site infection in women undergoing hysterectomy for benign gynecologic disease: a multicenter analysis using the national surgical quality improvement program data. J Minim Invasive Gynecol 2014;21:901-9.

40. Watanabe M, Suzuki H, Nomura S, et al. Risk factors for surgical site infection in emergency colorectal surgery: a retrospective analysis. Surg Infect (Larchmt) 2014;15:256-61. 
Supplementary

Table S1 One hundred and forty-four isolated microorganisms among 136 OSSI patients

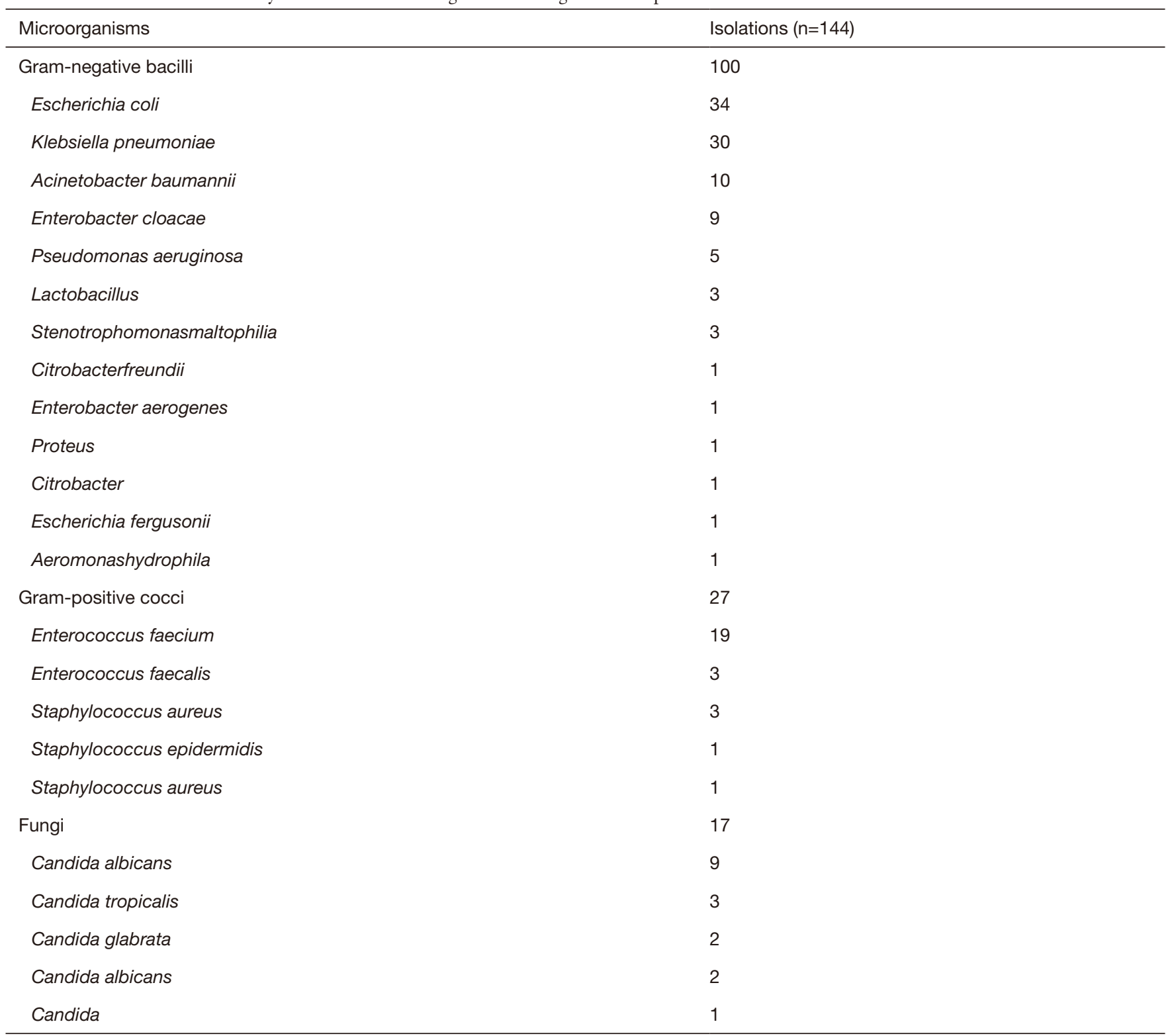

OSSI, organ/space surgical site infections. 


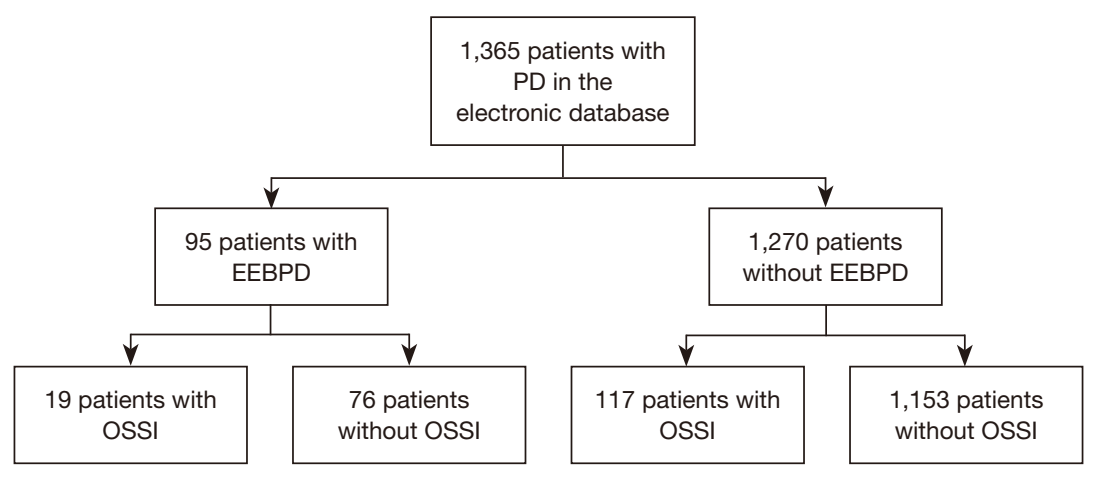

Figure S1 Study flowchart. PD, pancreaticoduodenectomy; EEBPD, ERCP exposure before PD; ERCP, endoscopic retrograde cholangiopancreatography; OSSI, organ/space surgical site infections. 